\title{
Principais características da Síndrome de Alagille: revisão narrativa
}

\author{
Main features of Alagille Syndrome: narrative review
}

Características principales del síndrome de Alagille: revisión narrativa

Nathália Guedes Carrijo ${ }^{1 *}$, Luciana Maria Carmona Mendes ${ }^{1}$, Mariana Sanches ${ }^{1}$, Thais Martins Oliveira ${ }^{1}$.

\begin{abstract}
RESUMO
Objetivo: Analisar os dados mais atuais e fidedignos para elucidar o quadro clínico, diagnóstico e tratamento da síndrome de Alagille. Revisão bibliográfica: A Síndrome de Alagille é uma doença multissistêmica, autossômica, dominante, com expressão variada, manifestada devido a defeitos no receptor NOTCH em sua via de sinalização, sendo 97\% dos casos causados por haploinsuficiência do gene JAGGED 1. A principal característica dessa síndrome é a hipoplasia do ducto biliar, tornando-o conhecida como displasia artériohepática. Ela também pode estar relacionada a malformações cardíacas, oftalmológicas e hepáticas. Atualmente é possível fazer o diagnóstico clínico através de critérios primários e secundários. $O$ tratamento ainda é incerto e as medidas tomadas estão relacionadas à progressão da sintomatologia, tornando o prognóstico relacionado com cada órgão afetado e apresentando uma mortalidade que varia de 10 a $20 \%$. Considerações finais: Nesta revisão, fornecemos um quadro mais abrangente relacionado à síndrome de Alagille por apresentar um desafio para classe médica pois, como visto nos artigos, é uma doença multissistêmica, e como há uma variedade no genótipo e fenótipo relacionado com a doença, o diagnóstico e a terapêutica da síndrome se tornam difíceis de serem elucidados.
\end{abstract}

Palavras-chaves: Síndrome de Alagille, Mutação, Anormalidades cardiovasculares, Colestase intra-hepática.

\begin{abstract}
Objective: To analyze the most current and reliable data to elucidate the clinical picture, diagnosis, and treatment of Alagille syndrome. Bibliographic review: Alagille Syndrome is a multisystemic, autosomal, dominant disease, with varied expression, manifested due to defects in the NOTCH receptor in it signaling pathway, with $97 \%$ of cases caused by haploinsufficiency of the JAGGED1 gene. The main feature of this syndrome is bile duct hypoplasia, making it known as arteriohepatic dysplasia. It can also be related to cardiac, ophthalmological, and liver malformations. Currently, it is possible to make a clinical diagnosis using primary and secondary criteria. The treatment is still uncertain, and the measures taken are related to the progression of the symptoms, making the prognosis related to each affected organ and presenting a mortality ranging from 10 to $20 \%$. Final considerations: In this review, we provide a broader picture related to Alagille syndrome as it presents a challenge for the medical profession because, as seen in the articles, it is a multisystem disease, and as there is a variety in the genotype and phenomenon related to the disease, the diagnosis and the differentiation of the same becomes determined to be elucidated.
\end{abstract}

Keywords: Alagille syndrome, Mutation, Cardiovascular abnormalities, Intrahepatic cholestasis.

\section{RESUMEN}

Objetivo: Analizar los datos más actuales y fiables para dilucidar el cuadro clínico, diagnóstico y tratamiento del síndrome de Alagille. Revisión bibliográfica: Él Síndrome de Alagille es una efermedad multisistémica, autosómica, dominante, de expresión variada, que se manifiesta por defectos en el receptor NOTCH en su vía de señalización, siendo el $97 \%$ de los casos por haploinsuficiencia del gen JAGGED 1. La principal característica de la síndrome es la bilis hipoplasia del conducto, por lo que se conoce como displasia arteriohepática. También puede estar relacionado con malformaciones cardíacas, oftalmológicas y hepáticas. Actualmente, es posible realizar un diagnóstico clínico utilizando criterios primarios y secundarios. El tratamiento aún es incierto, y las medidas tomadas están relacionadas con la progresión de los síntomas, haciendo que el prognóstico esté relacionado con cada órgano afectado y presentando una mortalidad que

1 Universidade de Franca (UNIFRAN), Franca - SP. *E-mail: nathaliaguedscarrijo@gmail.com 
oscila entre el 10 y el 20\%. Consideraciones finales: En esta revisión, brindamos un panorama más amplio relacionado con el síndrome de Alagille ya que presenta un desafío para la profesión médica porque, como se ve en los artículos, es una enfermedad multisistémica, y como existe una variedad en el genotipo y fenómeno relacionado con la enfermedad, el diagnóstico y la diferenciación de esta se determina a dilucidar.

Palabras clave: Síndrome de Alagille, Mutación, Anomalías cardiovasculares, Colestasis intrahepática.

\section{INTRODUÇÃO}

A síndrome de Alagille é também conhecida como displasia arterio-hepática ou síndrome de AlagilleWatson. Foi descrita inicialmente em 1969, por Alagille, sendo posteriormente referida por Watson e Miller no ano de 1973. Contudo, os critérios diagnósticos foram estabelecidos somente em 1975 por Alagille (DINIZ BL, et al., 2018)

Trata-se de uma doença multissistêmica autossômica dominante com expressão variada, gerada por defeitos do receptor Notch em sua via de sinalização. Aproximadamente $97 \%$ dos casos são causados por haploinsuficiência do gene JAGGED1 - JAG1 no braço curto do cromossomo 20, com locus 20p12, majoritariamente em consequência de mutações ou deleções no locus. Na maioria dos casos se trata de uma mutação "de novo", ou seja, quando as mutações se manifestam em um indivíduo, sendo inexistente nos genes de seus progenitores (CIOCCA M e ÁLVAREZ F, 2012; BRAVO A e ASTUDILLO C, 2015; LA SALLE SP, et al., 2015; MARTíNEZ AM, et al., 2012; LORENZO-ZÚÑIGA V, 2003).

Em menos de 1\% é causada devido a mutações no gene NOTCH2, com um locus em 1p13-p11, sendo associada a malformações renais. Além disso, é importante destacar que o gene JAGGED1 é responsável pela variabilidade dos achados fenotípicos presentes na síndrome. Esse gene codifica a síntese de uma proteína transmembranar que se liga ao receptor $\mathrm{NOTCH}$, cujo principal local de expressão é o sistema cardiovascular, que condiciona angiogênese e ductopenia anormais. Portanto, qualquer modificação nessa via de sinalização poderia acarretar desde pequenas alterações a até mesmo malformações e perda da homeostase, podendo envolver diferentes órgãos e sistemas (AROCENA E, et al., 2010; MARQUES SA, et al., 2008; CARBONELL BA, 2014).

Dessa forma, as manifestações clínicas são extremamente variáveis. Sua principal característica é a hipoplasia do ducto biliar, que pode estar relacionada a outras malformações, como distúrbios cardiológicos, presente em mais de $90 \%$ dos pacientes, oftalmológicos, renais, vertebrais, podendo ter grande variabilidade fenotípica em sua expressão, variando de formas subclínicas a graves expressões cardíacas, hepáticas ou renais. A falta de ducto biliar interlobular causa colestase crônica em 91\% dos pacientes (ORTEGA PÉREZ SN, et al., 2017; LA SALLE SP, et al., 2015).

Se tratando de manifestações hepáticas, pode haver hepatoesplenomegalia, hiperbilirrubinemia conjugada, hipercolesterolemia, hipertrigliceridemia e aumento de ácidos biliares e enzimas hepáticas. Além disso, alguns pacientes podem apresentar retardo de crescimento, prurido e xantomas (CIOCCA M e ÁLVAREZ F, 2012).

A estenose pulmonar periférica é a cardiopatia congênita mais frequente, representando $67 \%$ dos casos. Outras malformações são: tetralogia de Fallot (16\%), comunicação interventricular, comunicação interatrial, estenose aórtica e coarctação da aorta (CIOCCA M e ÁLVAREZ F, 2012, SARASÚA GL, et al., 2016).

Outro aparelho afetado seria o sistema renal, com acometimento de 20 a $73 \%$ dos casos de Alagille com displasia renal, acidose tubular renal, refluxo vesico ureteral e obstrução urinária. Foi observado que uma progressão para doença renal crônica e transplante renal são raros se associados com a síndrome (AYOUB MD e KAMATH BM, 2020).

Embriotóxon posterior acomete de 78-89\% dos casos; manifestações esqueléticas, como as vértebras em "asas de borboletas" em $80 \%$, vistas na radiografia anteroposterior; manifestações dismórficas leves, como fronte proeminentes, hipertelorismo moderado, nariz reto com ponta bulbosa, orelhas grandes, mandíbula proeminente, mento triangular, entre outros. Manifestações renais, como: rins pequenos e ecogênicos, cistos, obstrução ureteropélvica, nefropatia tubulointersticial e mesangiolipidose também foram descritas na síndrome (BRAVO A e ASTUDILLO C, 2015; CIOCCA M e ÁLVAREZ F, 2012; GUERRA YB, et al., 2013). 
Anormalidades vasculares são disseminadas e podem estar associadas à angiogênese alterada e à estrutura tecidual do sistema vascular. Há evidências que comprovam que a constituição de ductos biliares maduros está relacionada à formação da rede arterial intra-hepática. Ressalta-se que os genes envolvidos, JAGGED1 e NOTCH2, são constituintes da via de sinalização NOTCH, desempenhando papel importante na angiogênese (CIOCCA M e ÁLVAREZ F, 2012; COUCEIRO J, et al., 2014; GIRBES AP, et al., 2017).

A pseudo-obstrução ou obstrução prolongada dos ductos biliares ocasiona a agregação de colesterol no soro, sendo principalmente colesterol não esterificado, que fomenta a formação de uma lipoproteína. Dessa forma, a hipercolesterolemia de forma prolongada resulta na constituição de xantomas planos, tuberosos, xantelasmas, podendo ser confundidos com outros quadros dermatológicos. O acúmulo de bile resulta em um aumento nos pruritógenos plasmáticos, acarretando um desconforto no paciente, sendo inclusive um critério para o transplante de fígado em situações específicas. Além disso, outras manifestações como xerose, escoriações, rede venosa colateral, telangiectasias, eritema palmar, linfedema, ceratoderma e alopecia também foram referidas na síndrome (MARTíNEZ AM, et al., 2012).

Estudos recentes demonstram que $28 \%$ dos pacientes com síndrome de Alagille irão apresentar fraturas ósseas (AYOUB MD, KAMATH BM, 2020). Atualmente é possível realizar o diagnóstico clínico, tendo em vista a grande variedade de manifestações clínicas presentes. Anteriormente, para se fazer o diagnóstico era necessário a biópsia para confirmação, descartada hodiernamente. Dessa forma, a partir da clínica, pode-se dividir em dois critérios: os principais e os secundários, sendo posteriormente classificados na forma completa ou incompleta da síndrome (MARTíNEZ AM, et al., 2012; COSME Á, 2008).

A biópsia hepática verifica a escassez ou diminuição dos condutos biliares interlobulares. Em um lóbulo hepático normal encontra-se em cada tríade portal um espaço porta, uma veia, uma artéria e um dos condutos biliares interlobulares. Com isso, a relação normal entre o número de espaço porta e de conduto biliares deve oscilar entre 0,8 e 1,8. Na síndrome de Alagille, essa relação está diminuída, sendo menor que 0,4 (SEPÚLVEDA-HINCAPIÉ EM, et al., 2012)

O diagnóstico diferencial deve ser feito buscando infecções, doenças genético-metabólicas, atrésia biliar e causas idiopáticas. A pobreza dos ductos biliares observada na maioria dos pacientes com síndrome de Alagille também foi descrita em outros grupos de pacientes, como síndrome de Down, deficiência de alfa 1antitripsina, fibrose cística, infecções congênitas - citomegalovírus, rubéola, sífilis, e síndrome de Zellweger, portanto devem ser investigadas e descartadas. É muito extensa a quantidade de doenças que podem cursar com o quadro de colestase, portanto deve-se incluir também anormalidades estruturais, extra-hepáticas e intra-hepáticas, que causam obstrução ao fluxo biliar, causas tóxicas, infecciosas e metabólicas que modificam a síntese e excreção dos sais biliares (CIOCCA M e ÁLVAREZ F, 2012)

O alívio e tratamento do quadro de colestase, utiliza-se ácido ursodesoxicólico, colestiramina, rifampicina e fenobarbital. Com isso, há melhora da qualidade de vida em conjunto a um tratamento conservador da pele, que deve sempre ser hidratada a fim de reduzir sintomas de prurido e xantomas. Além disso, a devida importância deve ser dada para o suporte nutricional, sendo incluído dietas ricas em triglicerídeos de cadeia média e vitaminas lipossolúveis, devido à má absorção. Em pacientes com cirrose e insuficiência hepática ou prurido incoercível é indicado o transplante hepático. Há técnicas invasivas não cirúrgicas eficazes e técnicas cirúrgicas padrão para o manejo do comprometimento cardíaco, sendo individualizadas de acordo com o quadro de cada paciente (ORTEGA PÉREZ SN, et al., 2017; ORTIZ LM e SAMUDIO GC, 2015; CIOCCA M, ÁLVAREZ F, 2012, MARÍN URUEÑA SI, et al., 2017)

O prognóstico está relacionado com cada órgão afetado, como envolvimento hepático, transplante hepático, cardiopatia congênita, malformações renais, alterações vasculares e hemorragia cerebral. A gravidade das doenças cardíacas está associada diretamente com a mortalidade, sendo responsável por cerca de $15 \%$ dos falecimentos. O envolvimento hepático pode ter um curso benigno ou evoluir para cirrose em 14\% dos casos, sendo a principal causa de morbidade (ORTEGA PÉREZ SN, et al., 2017; GUERRA YB, et al., 2013).

Alguns fatores que indicam o transplante hepático são: falha lenta e progressiva da função hepática, desnutrição proteica energética grave, aparecimento de hipertensão portal e comprometimento significativo 
da qualidade de vida provocada pelo prurido refratário ao tratamento. O manejo dos pacientes com Síndrome de Alagille deve se concentrar na correção de complicações dos sistemas afetados, visando, portanto, um tratamento multidisciplinar. De modo geral, a mortalidade varia de 10 a 20\% (MARTíNEZ AM, et al., 2012; GUERRA YB, et al., 2013 SEPÚLVEDA-HINCAPIÉ EM, et al., 2012; COSME Á, 2008).

Isto posto, a presente revisão tem como objetivo analisar os dados mais atuais e fidedignos para elucidar o quadro clínico, diagnóstico e tratamento da síndrome de Alagille, tendo em vista que um melhor entendimento da situação clínica, o diagnóstico precoce, a correção das complicações e o tratamento multidisciplinar permitem um melhor prognóstico da criança com a síndrome.

\section{REVISÃO BIBLIOGRÁFICA}

Com base nos artigos revisados, observa-se que os autores afirmam que a síndrome de Alagille apresenta uma ampla variedade de apresentações clínicas. De forma resumida, pode-se dizer que as 5 características primordiais, não necessariamente todas concomitantes, a qual a síndrome se baseia são: (1) colestase intrahepática crônica; (2) embriotóxon posterior; (3) anormalidades cardiovasculares; (4) vértebras borboleta; (5) fácies peculiares. Sendo, portanto, um quadro rico de manifestações de vários órgãos e sistemas (DI PINTO D, ADRAGNA M, 2018; GONZÁLEZ PS, et al., 2015; CIOCCA M e ÁLVAREZ F, 2012).

Nota-se que o envolvimento hepático é comumente marcado por colestase crônica, sobretudo durante o período neonatal (CIOCCA M e ÁLVAREZ F, 2012; GONZÁLEZ PS, et al., 2015). Há também associação com hepatoesplenomegalia, dislipidemias e hiperbilirrubinemia conjugada, bem como acréscimo de ácidos biliares e de aminotransferases (LA SALLE SP, et al., 2015). Vários dos estudos sugerem estreita relação entre a síndrome de Alagille e o carcinoma hepatocelular, influenciando na morbimortalidade (MARÍN URUEÑA SI, et al., 2017; TORRES CP, et al., 2006). Observa-se também que a esplenomegalia é um achado incomum no início da doença, porém com a progressão da idade isso pode representar hipertensão portal e fibrose em $70 \%$ dos pacientes. A insuficiência pancreática também é descrita, mas sua avaliação é difícil devido à esteatorréia secundária à colestase (AYOUB MD e KAMATH BM. 2020)

O quesito dermatológico é determinado pela condição hepática, sendo os xantomas cutâneos evidenciados em paciente com colestase crônica quando a concentração de colesterol é superior a $500 \mathrm{mg} / \mathrm{dl}$. Os xantomas aumentam em número durante os primeiros anos de vida, mas podem regredir com o passar do tempo e a melhora da colestase. Quando presentes podem se localizar em diversas regiões do corpo, como: nuca, orelhas, superfície extensora dos dedos, dobras palmares, dobras inguinais, nádegas e fossa poplítea. Outra característica corresponde ao prurido que é geralmente incapacitante, ocorrendo a partir do segundo semestre de vida (CIOCCA M e ÁLVAREZ F, 2012; TORRES CP, et al., 2006).

O embriotóxon posterior configura uma faixa branca proeminente, localizada no ângulo da câmara anterior do olho, podendo vir associado a outras patologias, tais quais: câmara anterior superficial, ceratopatia em banda, catarata, microcórnea, ceratocone, exotropia e distrofia macular congênita (CIOCCA M e ÁLVAREZ F, 2012; GONZÁLEZ PS, et al., 2015).

As anormalidades cardiovasculares estão presentes em mais de $90 \%$ das crianças e se percebe pelos estudos que a mais frequente é a estenose da artéria pulmonar, a qual pode acarretar à hipertensão pulmonar e insuficiência a longo prazo do coração direito. A tetralogia de Fallot, a estenose aórtica, a coarctação da aorta, a comunicação interatrial e a interventricular também podem ser identificadas. É visualizado que o indicador mais significativo de mortalidade precoce no paciente com síndrome de Alagille é a presença de uma cardiopatia complexa (GONZÁLEZ PS, et al., 2015; GUERRA YB, et al., 2013; SARASÚA GL, et al., 2016).

As anormalidades na fusão do arco vertebral anterior são denominadas de vértebras de "asa de borboleta" e estão presentes na maioria dos pacientes com a síndrome, sendo possível a visualização por meio radiografia anteroposterior. Vale ressaltar que na síndrome de Alagille as "asas de borboleta" têm aparência simétrica como forma de ajudar a diferenciar, visto que as anormalidades da segmentação vertebral ocorrem em várias outras doenças. Há outras manifestações esqueléticas menos frequentes, como: fusão da vértebra 
adjacente, hemivértebra, ausência da décima segunda costela, espinha bífida oculta, afinamento da distância interpeduncular da coluna lombar, bem com casos de encurtamento das falanges distais dos dedos que conferem aparência fusiforme, craniossinostose e sinostose radiocubital (BRAVO A e ASTUDILLO C, 2015; CARBONELL BA, 2014; COSME Á, 2008).

$\mathrm{Na}$ fascie, o paciente pode apresentar um aspecto singular, com olhos fundos com hipertelorismo moderado, fissuras oblíquas palpebrais ascendentes, nariz reto com ponta bulbosa, ponte nasal deprimida, orelhas volumosas, fronte e mandíbula sobressalentes, aliadas a um queixo pontudo (GUERRA YB, et al., 2013; LA SALLE SP, et al., 2015; COSME Á, 2008). Menos comumente, o paciente com AS pode apresentar envolvimento vascular (podendo ocasionar AVC e riscos de sangramento), renal (com alterações estruturais e funcionais), pancreático, déficit de crescimento, neurológico e na aprendizagem (DI PINTO D e ADRAGNA M, 2018; AROCENA E, et al., 2010; ESPINOSA VL, et al., 2010). Foi descrita também uma possível associação com pseudo-hermafroditismo (GELONCH RM, 2004).

Destarte, para estabelecermos o diagnóstico, existem os critérios primários e os critérios secundários. (Quadro 1).

Quadro 1 - Critérios primários e secundários.

\begin{tabular}{|l|l|}
\hline \multicolumn{1}{|c|}{ Critérios primários } & \multicolumn{1}{c|}{ Critérios Secundários } \\
\hline Colestase crônica & Xantomas \\
\hline Fáscies dismórficas & Retardo de crescimento \\
\hline Anomalias cardiovasculares & Infecções pulmonares recorrentes \\
\hline Defeitos dos arcos vertebrais & Insuficiência pancreática \\
\hline Embriotóxon posterior & Hipotireoidismo \\
\hline & Anormalidades vasculares \\
\hline & Anormalidades na voz \\
\hline & Anormalidades neurológicas \\
\hline & Hipogonadismo e puberdade tardios \\
\hline & Retardo mental \\
\hline & Anormalidades Renais \\
\hline
\end{tabular}

Fonte: Carrijo NG, et al., 2021; dados extraídos de Ortega SN, et al., 2017.

De acordo com esses critérios, podemos considerar duas formas: se houver os cinco primários presentes, é considerada a forma completa; se houver pelo menos três critérios primários (entre os quais está a colestase), é considerada a forma incompleta. Quando há histórico familiar evidente da síndrome de Alagille, os critérios mudam, limitando-se a apenas 2 critérios. Atualmente a biópsia não é necessária para estabelecer o diagnóstico quando há colestase juntamente com os demais critérios primários. Caso seja feita o estudo patológico através da biópsia, os achados histológicos evidenciam uma redução na relação entre o número de ductos biliares e dos espaços-porta, resultando em uma relação menor que 0,4 em pacientes portadores da síndrome, sendo que em pacientes sem a comorbidade os valores variam entre 0,9 e 1,8 (ORTEGA PÉREZ SN, et al., 2017; CIOCCA M e ÁLVAREZ F, 2012; MARTíNEZ AM, et al., 2012; COSME Á, 2008; SEPÚLVEDA-HINCAPIÉ EM, et al., 2012).

Em relação à ultrassonografia hepática, pode-se encontrar uma aparência nodular, visto que há uma distribuição heterogênea deficiente dos ductos biliares interlobulares no fígado, causando dessa forma áreas de atrofia em regiões sem o ducto biliar e hipertrofia nos locais onde há o ducto. Tais nódulos com tecido hepático sem alterações podem explicar a melhora da colestase, que geralmente exacerba até à idade escolar, e em algumas crianças convalesce ou permanece estável (CIOCCA M, ÁLVAREZ F. 2012)

Devido à baixa expressão do fígado e à sobreposição de características clínicas e resultados de exames histológicos, notamos que é difícil diagnosticar diferencialmente o processo que causa a colestase neste período de vida. Ademais, nas primeiras semanas de vida, as características faciais que tornam o diagnóstico da Síndrome suspeito, juntamente com a colestase, nem sempre são nítidas (ORTIZ LM e SAMUDIO GC, 2015; CIOCCA M e ÁLVAREZ F, 2012) 
Além disso, as doenças colestáticas têm uma ampla ocorrência, incluindo: anormalidades estruturais, extra-hepáticas e intra-hepáticas, que podem causar obstrução do fluxo biliar; causas infecciosas, tóxicas ou metabólicas que podem alterar o mecanismo de síntese e excreção dos sais biliares. Devemos levar em conta que a proliferação ductal com inflamação portal pode ser observada em neonatos, levando ao diagnóstico incorreto de atresia biliar. Dessa forma, deve-se sempre ser descartadas outras hipóteses diagnósticas que podem cursar com manifestações clínicas semelhantes (ORTIZ LM e SAMUDIO GC, 2015; CIOCCA M e ÁLVAREZ F, 2012; JARA PALOMA, 2010).

Por outro lado, quando há a presença de estenose pulmonar periférica, deve-se realizar diagnóstico diferencial com a Síndrome de Williams (SW), patologia na qual a estenose também está associada, além de cursar similarmente com alterações da face - face de gnomo ou fadinha, alterações do desenvolvimento motor e cognitivo, além de anomalias renais e cardiovasculares. Excetuando-se essas alterações, as demais alterações são distintas. Além do mais, a SW não é transmitida geneticamente, sendo uma rara desordem genética (CIOCCA M e ÁLVAREZ F, 2012; ASHKAN LASHKARI BS, et al., 1999).

Em referência às manifestações oftalmológicas, a acuidade visual é usualmente sem alterações. Entretanto, recomenda-se de rotina o exame oftalmológico para verificar a progressão desse comprometimento e para identificar o glaucoma em intervalo hábil. Foi identificado que menos de $1 \%$ das crianças portadoras da síndrome podem evoluir com perda visual progressiva e ocasionalmente cegueira permanente, possivelmente secundária a anomalias vasculares (CIOCCA M e ÁLVAREZ F, 2012).

Percebe-se que em alguns casos de extrema evolução da doença, algumas condutas visam evitar a progressão da sintomatologia e complicações que essa patologia apresenta. Um exemplo é o transplante hepático utilizado principalmente para tratamento de doenças hepáticas crônicas já em fase de descompensação irreversível. Ou seja, geralmente é recomendado quando não se consegue mais controlar a colestase com um tratamento médico adequado e surgem, concomitantemente, algumas alterações secundárias como prurido, hipertensão portal, cirrose e ascite. No entanto, a decisão final é baseada principalmente em complicações, como trombose e sangramento do pré-transplante e pós-transplante, já que a SA apresenta um acometimento de natureza multissistêmica (ORTEGA PÉREZ SN, et al., 2017; CIOCCA M e ÁLVAREZ F, 2012).

Algumas manifestações hepáticas apresentam variações em sua apresentação, indo desde uma icterícia leve até prurido intenso, de acordo com a evolução da deterioração hepática. A realização de um transplante hepático como forma de tratamento traz uma regressão completa de alguns sintomas, como o prurido, considerado um dos piores sintomas na doença hepática crônica, além da normalização da função hepática, redução de lesões cutâneas e dos níveis de lipidograma (SEPÚLVEDA-HINCAPIÉ EM, et al., 2012).

Quando não se torna possível a realização de um transplante hepático o tratamento do prurido se estabelece através de medidas conservadoras como utilização de produtos emolientes para hidratação da pele, corte de unha e banhos curtos, evitando o atrito na pele com toalhas de banho. Os anti-histamínicos podem também proporcionar alívio sintomático leve, assim como a Rifampicina que apresenta uma boa resposta em relação a essa sintomatologia (CIOCCA M e ÁLVAREZ F, 2012).

Foi notado também que a SA é uma das principais causas de colestase genética, ocorrendo devido uma alteração no fluido biliar, e trazendo como consequência a retenção de sais biliares, causando danos celular ao nível do fígado, além de um regurgitamento no sangue dos produtos da bile. Com isso, ocorre uma diminuição do fluxo da bile a nível intestinal, tornando a qualidade desse fluido inadequada, já que não contém concentrações de micelas suficientes para transporte de sais biliares e vitaminas solúveis em gordura. Toda essa patogenia pode ocasionar uma má digestão de gordura, ocasionando esteatorreia e má digestão de proteínas, ocasionando uma desnutrição (MARÍN URUEÑA SI, et al., 2017; MARQUES SA, et al., 2008; JARA PALOMA, 2010).

Com isso, como tratamento dessa colestase, temos que instituir uma dieta proteica com proteínas e gorduras hidrolisadas do tipo triglicerídeos de cadeia média, já que não exigem que sais biliares sejam absorvidos, além de uma sonda nasogástrica ou gastrostomia, garantindo assim todos os suprimentos necessários, exceto em casos de cirrose e hipertensão portal, no qual a gastrostomia está contraindicada (JARA PALOMA, 2010; ORTIZ LM e SAMUDIO GC, 2015). 
Algumas literaturas também trazem como opção de tratamento o ácido ursodesoxicólico, colestiramina, rifampicina e naltrexona para melhora do prurido intenso e xantoma. Adicionado a isso, para evitar a desnutrição, suplementa-se vitaminas como cálcio e zinco (ORTIZ LM e SAMUDIO GC, 2015; MARÍN URUEÑA SI, et al., 2017; JARA PALOMA, 2010). Nos casos de hipercolesterolemia o paciente não requer tratamento dietético ou medicamentoso, sendo as estatinas reservadas para os casos com xantomas debilitantes. (CIOCCA M e ÁLVAREZ F. 2012)

Uma opção cirúrgica sem ser o transplante hepático também observada é um desvio biliar externo, tendo tido repercussão positiva em pacientes com prurido e xantomas intratáveis, diferente daqueles que apresentam fibrose hepática na qual esse tipo de abordagem cirúrgica apresentou resultados fracos (MARQUES SA, et al., 2008; CIOCCA M e ÁLVAREZ F, 2012; JARA PALOMA, 2010).

Já para a sintomatologia cardiovascular, técnicas invasivas não cirúrgicas têm efeito positivo como terapêutica, e em alguns casos pode-se utilizar técnicas cirúrgica padrão, já que a resolução da cardiopatia congênita cianótica melhora a função hepática e reduz a colestase, assim como no tratamento do envolvimento renovascular sintomático, que pode ser solucionado com a utilização de um stent, e nos casos de acidose tubular renal, a melhora se dá com administração de bicarbonato (CIOCCA M e ÁLVAREZ F, 2012; ORTEGA PÉREZ SN, et al., 2017).

No prognóstico, observamos que um indicativo de grande agressividade da síndrome se encontra numa icterícia presente no período neonatal, se comportando como mau prognóstico a persistência prolongada da colestase, com um nível de bilirrubina $>5 \mathrm{mg} / \mathrm{dl}$. Além disso, depende fundamentalmente do envolvimento hepático, das manifestações cardiovasculares e renais (LORENZO-ZÚÑIGA V, 2003; MARTíNEZ AM, et al., 2012). A expectativa de vida aos 20 anos de acompanhamento notada foi de $60 \%$ em receptores de transplante (GUERRA YB, et al., 2013). Encontrando uma mortalidade de 10-20\% (ROMÁN CASTELLINI EV, et al., 2012).

\section{CONSIDERAÇÕES FINAIS}

Diante de uma revisão efetuada, observou-se que a Síndrome de Alagille é uma condição rara com ampla variedade de apresentação clínica, sendo cinco características primordiais, mas não concomitante. Um melhor entendimento da situação clínica, o diagnóstico precoce, a correção das complicações e o tratamento multidisciplinar permitem um melhor prognóstico da criança com a síndrome. Se trata de uma condição clínica que carece de informações e condutas, podendo ocasionar evoluções desfavoráveis e graves para o paciente. Devido a isso, percebe-se, então, a relevante necessidade de que haja novas pesquisas sobre o assunto a fim de esclarecer, de uma forma consistente e fidedigna, toda a história natural da doença.

\section{REFERÊNCIAS}

1. AROCENA E, et al. Síndrome de Alagille: A propósito de un caso. Arch. Pediatr. Urug, 2010; 81(3): 158-162.

2. ASHKAN LASHKARI BS, et al. Williams-Beuren Syndrome: An update and review for the primary Physician. Clinical Pediatrics, Clinical Pediatrics, 1999; 38: 189-208.

3. AYOUB MD, KAMATH BM. Alagille syndrome: Diagnostic challenges and advances in management. Pediatric Liver Diseases, 2020; 10(11): 907.

4. BIDAGUREN $A$, et al. Coriorretinopatía geográfica progresiva asociada al síndrome de Alagille. Arch Soc Esp Oftalmol., 2007; 82(8): 513-515.

5. BRAVO A, ASTUDILLO C. Estenosis aórtica severa en paciente con Síndrome de Alagille. Rev Chil Cardiol., 2015; 34: 214-219.

6. CARBONELL BA. Rol de la vía de señalización NOTCH durante el desarrollo de estructuras craneofaciales. Rev Fac Odontol Univ Antioq, 2014; 26(1): 164-179.

7. CIOCCA M, ÁLVAREZ F. Síndrome de Alagille. Arch Argent Pediatr., 2012; 110(6): 509-515.

8. COSME Á. Características clínicas y moleculares de una familia con síndrome de Alagille. Med Clin (Barc), 2008; 130(1): 17-19.

9. COUCEIRO J, et al. Síndrome de Alagille asociado a sinostosis radiocubital proximal: caso clínico y revisión de la literatura. Rev Esp Cir Ortop Traumatol., 2014; 60(1): 81-85.

10. DINIZ BL, et al. Aumento dos níveis de quitotriosidase em um paciente com síndrome de Alagille: associação ou coincidência?. Jornal Brasileiro de Patologia e Medicina Laboratorial, 2018; 54(1): 37-39. 
11. DI PINTO D, ADRAGNA M. Renal abnormalities in children with Alagille syndrome. Arch Argent Pediatr 2018; 116(2): $149-153$.

12. ESPINOSA VL, et al. Rehabilitación bucal bajo anestesia general en el síndrome de Alagille. Reporte de un caso. Rev. Odont. Mex, 2010; 14(1): 63-68.

13. GELONCH RM. Síndrome de Alagille asociado a seudohermafroditismo masculino. An Pediatr (Barc) 2004; 60(5): 479-87.

14. GIRBES AP, et al. Síndrome de Alagille: hipoplasia de canales semicirculares posteriores. Acta Otorrinolaringol Esp., 2017; 69(1): 58-59.

15. GONZÁLEZ PS, et al. Síndrome de Alagille asociado a atresia intestinal. Gastroenterol Hepatol. 2015.

16. GUERRA YB, et al. Síndrome de Alagille. Revista Cubana de Pediatría. 2013; 85(1): 130-136.

17. JARA PALOMA. Colestasis: enfoque actual. Revista Gastrohnup., 2010;12(1): S27-S30.

18. LA SALLE SP, et al. Tumor hepático com vías biliares normales en um paciente con síndrome de Alagille: a propósito de un caso. Arch Argent Pediatr., 2015; 113(4): e223-e225.

19. LORENZO-ZÚÑIGA V. Hipertensión portal presinusoidal por trombosis portal en una paciente con síndrome de Alagille. Gastroenterol Hepatol., 2003; 26(5): 294-6.

20. MARÍN URUEÑA SI, et al. Síndrome de Alagille con fenotipo atípico diagnosticado por estudio molecular: mutación no descrita en JAG1. Med Clin (Barc). 2017; 149(10):462.

21. MARQUES SA, et al. Você conhece esta síndrome?. An. Bras. Dermatol., 2008; 83(5): 476-479.

22. MARTÍNEZ AM, et al. Manifestaciones dermatológicas del síndrome de Alagille. Bol Med Hosp Infant Mex, 2012; 69(2): 139-143.

23. ORTEGA PÉREZ SN, et al. Síndrome de Alagille; una patología que tener en cuenta. Rev Pediatr Aten Primaria, 2017; 19: $267-70$.

24. ORTIZ LM, SAMUDIO GC. Síndrome de Allagile. Presentación de un caso. Pediatr. Asunción, 2015; 41(1): 51-56.

25. RAMIREZ NR, et al. Biopsia hepática percutánea, experiencia de ocho años en el servicio de gastroenterologia del Hospital de Niño "Dr. Ovidio Aliaga Uria". La Paz. Rev. bol. ped., 2008; 47(3): 155-159.

26. ROMÁN CASTELLINI EV, et al. Evolución de paciente pediátrico con Síndrome Alagille. Reporte de caso. Rev Méd Electrón, 2012; 34(2): 231-240.

27. SARASÚA GL, et al. Aterosclerosis calcificada del tronco de la arteria pulmonar, estenosis de las arterias pulmonares principales y dilatación postestenótica de arterias pulmonares segmentarias en un paciente con síndrome de Alagille. Arch Bronconeumol., 2016; 53(2): 73-74.

28. SEPÚLVEDA-HINCAPIÉ EM, et al. Síndrome de alagille experiencia clínica de catorce casos en medellín, Colombia. Revista gastrohnup., 2012; 14(2): 49-54.

29. TORRES CP, et al. Síndrome de Alagille: relato de caso. Revista Instituto Ciência Saúde, 2006; 24(2).

30. VÁZQUEZ RF, et al. Síndrome de Alagille: una causa de colestasis intrahepática persistente. Bol. Med. Hosp. Infant. Mex., 2009; 66(2): 178-188. 